\title{
Effect of Stress on Preterm Labour- A Case Control Study in a Tertiary Care Centre
}

\author{
Authors \\ Dr Shaila $S^{1}$, Dr Serbin Mohammed ${ }^{2}$, Dr Jesna Hassan ${ }^{3}$, Dr Sithara $S^{4}$ \\ ${ }^{1}$ Professor Dept. of O and G, SAT Hospital, Govt. Medical College, Trivandrum \\ ${ }^{2}$ Asst. Professor, Dept. of Surgery, Govt. Medical College, Trivandrum \\ ${ }^{3}$ Post Graduate Student, Dept. of O and G, SAT Hospital, Govt. Medical College, Trivandrum \\ ${ }^{4}$ Asst. Surgeon, Family Planning, Dept. of O and G, SAT Hospital, Govt. Medical College, Trivandrum \\ Corresponding Author \\ Dr Shaila S \\ Professor Dept. of O and G, SAT Hospital, Govt. Medical College, Trivandrum
}

\section{Introduction}

Preterm delivery is one of the most challenging obstetric complication encountered in day to day practice and is the most vulnerable of all complications to manage for paediatricians. It is the single greatest cause of perinatal mortality and morbidity worldwide. Preterm delivery is defined as spontaneous expulsion of the products of conception after viability and before 37 completed weeks. More than 1 million infants die each year because they are born too early according to the just released WHITE PAPER, the Global and Regional Toll of Preterm birth.

It is the leading cause of infant mortality and morbidity; its prevalence in our population is unacceptably high and has not decreased over the last 40 years; and its etiology is unknown in a substantial proportion of cases. The ongoing search to better elucidate its underlying causes and pathophysiological mechanisms has identified maternal stress as a variable of interest. The question of the role of stress in preterm birth is, however, complex and challenging for many reasons. First, the basic physiological and pathophysiological mechanisms that underlie the timing of onset of human parturition and preterm birth, respectively, are not yet well understood. Second, the study of stress processes in pregnancy is complicated by the effects that pregnancyrelated alterations in maternal physiology produce on central and peripheral systems implicated in the experience of and psychobiological responses to stress.

Strategies to prevent preterm delivery have focused on early diagnosis of preterm labour. Diagnosing early preterm labour is difficult and has a high false positive rate and it may result in unnecessary and potentially hazardous treatment for thousands of women. Premature babies are at risk of developing long term complications like cerebral palsy, vision and hearing loss and mental retardation. 


\section{Psychological stressors}

The mechanisms involved in the psychosocial associations of preterm birth are not well understood. Research on psychosocial factors and preterm birth has accumulated rapidly in recent years. Some psychosocial factors implicated in the etiology of preterm birth include major life events, chronic stress, maternal anxiety, personal racism, and lack of support (Behrman \& Butler, 2007).

Moreover, women who fail to show the expected decrease in generalized stress and anxiety or dampening in the cortisol awakening response during pregnancy are at increased risk for preterm delivery (Buss et al., 2009b; Glynn et al., 2008).

Evidence suggests that the normal trajectory of placental CORTICOTROPIN RELEASING HORMONE production over the course of gestation may be accelerated by an adverse intrauterine environment characterized byphysiological stress. For example, elevated placental CORTICOTROPIN RELEASING HORMONE has been observed in pregnancies complicated by pre-eclampsia, reduced uteroplacental perfusion, intrauterine infection, and in cases where fetal distress has led to elective preterm delivery (Giles et al., 1996). A series of in vitro studies (Petraglia et al., 1987; Petraglia et al., 1989; Petraglia et al., 1990) have shown that CORTICOTROPIN RELEASING HORMONE is released from cultured human placental cells in a dose-response manner in response to all the major biological effectors of stress, including cortisol, catecholamines, and pro-inflammatory cytokines.

Major life event

Exposure to severe life events has been linked to very and extremely pre-term births.Khashan ${ }^{76}$ and colleagues carried out a study evaluating the influence of exposure to severe life events and preterm, very preterm and extremely preterm birth. Exposures were definedas death or serious illness in close relatives 6 months prior to conception or in the first or second trimester of pregnancy. In their cohort of 1.35 million singleton live births, they found that maternal exposure to severe life events contributed significantly to the risk of preterm birth; severe life events in close relatives increased the preterm birth rate by $16 \%$, while if the severe life event involved an older child, the risk of preterm and very preterm birth was increased by $23 \%$ and $59 \%$ respectively (Khashan et al, 2009) ${ }^{76}$.

\section{Work related stressors}

With the increasing number of mothers who continue to work late into their pregnancy, occupational stress is hypothesized to be an important contributor to adverse reproductive outcomes, both for mother and baby (Mutambudzi et al., 2011) ${ }^{97}$. Physical stressors such as shift work, long hours standing, and heavy lifting have been consistently associated with increased risk of preterm birth (Gold et al 1994). Theoretically, having and being able to maintain a job, is an indicator in itself of a higher socioeconomic status, which has an inverse effect on preterm birth rates. Recent studies have found no increase in the rate of preterm birth with employment, but these are mainly studies carried out in developed countries, where physical strain and hazardous working conditions are not the norm.

\section{Behavioral stressors}

Behavioral stressors such as smoking, alcohol and illicit drug use, poor eating habits, sexual and physical activity, are of great importance as modifiable risk factors, as their elimination can lead to an effective reduction in the preterm birth rate. These behaviors pose specific challenges in establishing a cause and effect relationship because of their inherent complexity (Behrman \& Butler, 2007). In addition, unfavorable health activities tend to cluster: for example women with poor diets often have other potentially detrimental behaviors, such as a lack of physical activity, and vice versa (Behrman \& Butler, 2007 ${ }^{45}$ ). In the UK in 2004, 25\% of all adults were smokers (NHS). $17 \%$ of mothers continued to smoke throughout pregnancy, whilst $49 \%$ of smoking mothers gave up before or during pregnancy (NHS). Studies have shown that lower or stressful socioeconomic 
status contributes significantly to persisting to smoke during pregnancy (Weaver, 2007). Smoking is strongly related to placental abruption, reduced birth weight and infant mortality, however the relationship of cigarette smoking to preterm birth is somewhat modest and not completely replicable (Behrman \& Butler, 2007). Its influence on pregnancy outcomes, like preterm birth, is most notable in the third trimester and there is no increased risk detected in mothers who smoke prior to the onset, or in the early stages of pregnancy (Behrman \&Butler, 2007). High levels of alcohol use during pregnancy have detrimental effects on fetal development and subsequent neonatal survival. Women who have more than one drink a day are at an increased risk of preterm labour. According to a study carried out by Albertsen et al. 2004, the relative risk of preterm delivery and very preterm birth, <32 completed weeks, among women who had seven or more drinks a week during pregnancy was 1.77 and 3.26 respectively, compared to non-drinkers. If less than 4 units a week of alcohol was consumed, then there was no increased risk of preterm birth (Albertsen et al., 2004) ${ }^{32}$. Due the differing effects of alcohol on the fetus depending on the amount consumed and the limitations associated with self reporting, the relationship between alcohol and preterm birth remains unclear. Illicit drug use, especially cocaine, has been widely implicated as an important cause of preterm birth. Cocaine use in the United States rose from 3.2\% in 1972 to $12.5 \%$ in 1985, and continues to rise (Rouse, $1991)^{110}$. Antenatal cocaine users experience significantly increased risk of preterm birth compared with that for non-users, with an odds ratio of 3.38 (Gouin,2011). Marijuana smoking does not appear to significantly increase the risk of preterm labour. Its adverse effects come mainly from the inhalation of combustion material (Behrman \& Butler, 2007) ${ }^{45}$. Repeatedly, evidence shows that mothers who live a favorable lifestyle are at a reduced risk of adverse pregnancy outcome, including a reduction in the risk of preterm labour (Behrman\& Butler, 2007).
Continued efforts are required to better understand and therefore define the aspects of a positive lifestyle that are associated with a reduced risk of preterm birth.

\section{Methods}

Design: A case control study. There were 230 patients in control group and 230 patients taken as cases.

Duration and setting: The study was conducted from November $1^{\text {st }} 2012$ to November $30^{\text {th }} 2013$ at Sree Avittom Thirunal Hospital, Medical College Thiruvananthapuram.

\section{Inclusion Criteria}

1) Gestational age of 24-37 weeks was considered for this study. Gestational age was estimated by the patients' last menstrual period (LMP). It was determined on the basis of whether menstruation was regular or by ultrasonography detecting gestational age of $<20$ weeks.

2) Previous ultrasonogram showing normal fetal morphology.

\section{Exclusion Criteria}

1. IUDs and still births.

2. Unwilling women.

Sampling method: Consecutive cases satisfying the sample size and the succeeding normal delivery of each case is taken as control. All consecutive admissions were screened and the list of eligible cases was made and the units were selected accordingly.

\section{Sample size}

Sample is calculated using the formula

$$
\begin{aligned}
& \mathrm{n}=\left\{\mathrm{Z}_{1-\alpha / 2} 2 \sqrt{\mathrm{p}(1-\mathrm{P})+\mathrm{Z}_{1-\mathrm{p}} \mathrm{p} 1(1-\mathrm{p}){ }_{+}^{+\mathrm{p} 2(1-\mathrm{p} 2)}}\right\} \\
& \left(\mathrm{P}_{1}-\mathrm{P}_{2}\right)^{2} \\
& \mathrm{P}_{1}=\mathrm{ORxP}_{2} \\
& \mathrm{ORxP}_{2}\left(1-\mathrm{P}_{2}\right) \\
& \mathrm{P}=\mathrm{P}_{1}+\mathrm{P}_{2} \\
& 2
\end{aligned}
$$


$\mathrm{P} 2=$ proportion of given disease absent

$\mathrm{P} 1=$ proportion of given disease is present

$\mathrm{OR}=$ Odds ratio

$\alpha=$ Significance level

$1-\beta=$ Power

From literature

$\begin{array}{ll}\begin{array}{l}\text { Proportion of exposure in control group (Vaginal } \\ \text { infections) }\end{array} & 0.08 \\ \text { Anticipated odds ratio } & 2.4 \\ \text { Proportion of exposure in case group } & 0.17 \\ \text { Power (1- beta) \% } & 80 \\ \text { Alpha error (\%) } & 5 \\ 1 \text { or } 2 \text { sided } & 2\end{array}$

Required sample size in each of the case \& control groups

For the present study 230 case and controls were taken as sample size. All consecutive cases satisfying the inclusion and exclusion criteria was studied. For every preterm birth that occurred between gestational ages 24 -37 weeks while term babies were those whose delivery occurred at or beyond a gestation age of 37 but below 42 completed weeks.

\section{Data Analysis}

Qualitative variables were analysed using proportions and quantitative variables was expressed in mean and standard deviation. Collected data was entered in MS EXCEL. Completeness was checked and analysis was done using statistical software SPSS version 20. Chi square test, Fischer's exact test and logistic regression used for finding the risk factors. Appropriate statistical tests were used to interpret the results and derive summary statistics the distribution of variables was looked into and appropriate statistical significance was undertaken

\section{Results}

A total of 460 patients were studied, of 460 patients 230 patients under the cases are between $24-37$ weeks of gestation and 230 patients were taken as control are patients who delivered normally after 37 weeks but before 42 weeks.

Table : Distribution of maternal Stress among the Patients

\begin{tabular}{|l|c|c|c|c|c|c|}
\hline \multirow{2}{*}{ Maternal stress } & \multicolumn{2}{|c|}{ Case } & \multicolumn{2}{c|}{ Control } & \multicolumn{2}{c|}{ Total } \\
\cline { 2 - 7 } & $\mathrm{N}$ & $\%$ & $\mathrm{~N}$ & $\%$ & $\mathrm{~N}$ & $\%$ \\
\hline Severe & 11 & 4.8 & 3 & 1.3 & 14 & 3 \\
\hline Moderate & 33 & 14.3 & 20 & 8.7 & 53 & 11.5 \\
\hline Mild & 46 & 20 & 79 & 34.3 & 125 & 27.2 \\
\hline Nil & 140 & 60.9 & 128 & 55.7 & 268 & 58.3 \\
\hline Total & 230 & 100 & 230 & 100 & 460 & 100 \\
\hline$\chi^{2}=17.009$ & $d f=1$ & \multicolumn{9}{|c|}{$p .001$}
\end{tabular}

Mothers with moderate to severe stress are significantly more in the study group $(19.1 \%)$ than in the control group (10\%). Table 21 showed the different levels of stress and its distribution in both the groups. Comparing cases and controls a higher percentage of cases was seen to have no stress. The chi- square test showed an association between stress levels and preterm labour. This is once more significant .since severe and moderate stress is a significant maternal determinant in the etiology of preterm labour. $\mathrm{p}<0.001$

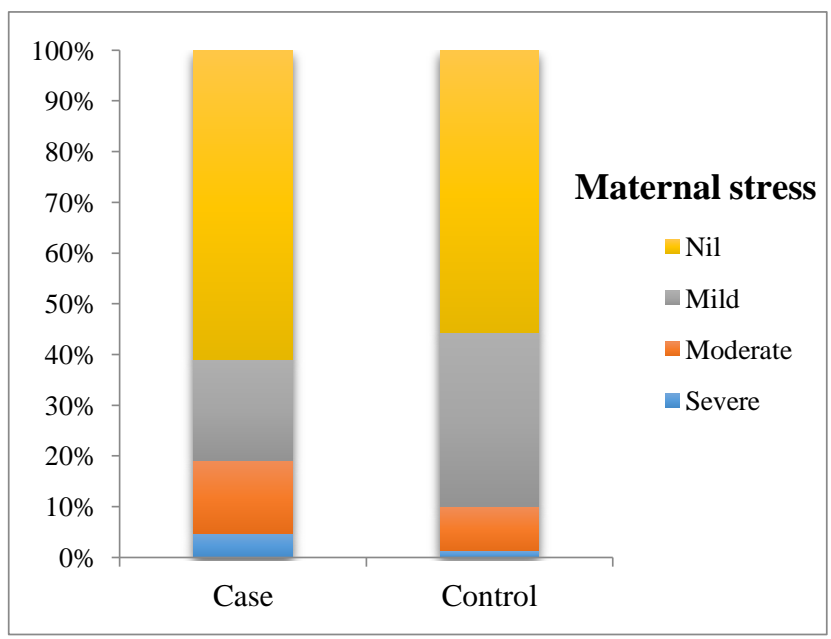




\section{Discussion}

Several studies have previously suggested that women who experience psychological or social stress during pregnancy are at significantly increased risk for shorter gestation, earlier onset of spontaneous labor, low birth weight infant $(<2500 \mathrm{~g})$ and preterm delivery ${ }^{[1-9]}$.

Stressful events can promote the secretion of hormones that are involved in the onset of delivery, and that play a role in the regulation of the birth process. In addition, stress reactions may impair the immunologic defense against microorganisms that may colonize the uterus and initiate uterine contractions or weaken the chorion. Consequently, the membranes may become vulnerable and at risk for (preterm) premature rupture, resulting in preterm birth. The problem is complex and requires a multifaceted approach to come to meaningful and realistic solutions. In addition, there is a need for more research in coping, resiliency and other stress management techniques during pregnancy ${ }^{[3]}$ Concepts in evolutionary biology and developental plasticity support a rationale for considering a role for maternal stress in preterm birth. Evidence from population-based epidemiological and clinical studies suggests that after accounting for the effects of other established sociodemographic, obstetric and behavioral risk factors, women reporting higher levels of psychological stress during pregnancy are at significantly increased risk of preterm birth. However, at the individual level, the specificity and sensitivity of maternal stress as a predictor of preterm birth risk is, at best, modest. ${ }^{[11]}$

Social support interventions need to be guided by predictive models and more needs to be done to elucidate which components of interventions account for the largest variability in birth outcomes. Lastly, more work can be done to evaluate how physiologic responses to stressors might account for health disparities.

\section{Conclusions}

Preterm birth negatively affect $s$ the newborn child, both in the short and long term. In conclusion, approximately $40 \%$ of the preterm births in this study were estimated to be due to maternal stress exposure during pregnancy as an attributable risk factor. Thus it is of great importance to identify and possibly alleviate the exposure to stress during pregnancy and by doing that try to decrease the preterm birth rate.

\section{References}

1. Federenko IS, Wadhwa PD. Women's mental health during pregnancy influences fetal and infant developmental and health outcomes. CNSSpectr. 2004;9:198-206.

2. Entringer S, Buss C, Wadhwa PD. Prenatal stress, development, health anddisease risk: a psychobiological perspective-2015 Curt Richter Award Paper. Psychoneuroendocrinology. 2015;62:366-75.

3. Wadhwa PD, Culhane JF, Rauh V, Barve SS. Stress and preterm birth: neuroendocrine, immune/inflammatory, and vascular mechanisms. Matern Child Health J. 2001;5:119-25.

4. Hoirisch-Clapauch S, Brenner B, Nardi AE. Adverse obstetric and neonataloutcomes in women with mental disorders. Thromb Res. 2015;135.Lilliecreutz et al. BMC Pregnancy and Childbirth (2016) 16:5 Page 7 of 8

5. Staneva A, Bogossian F, Pritchard M, Wittkowski A. The effects of maternal depression, anxiety, and perceived stress during pregnancy on preterm birth: a systematic review. Women Birth. 2015;28:179- 93.

6. Christian LM. Effects of stress and depression on inflammatory immuneparameters in pregnancy. Am $\mathrm{J}$ Obstet Gynecol. 2014;211:275- 7.

7. Glover V. Prenatal stress and its effects on the fetus and the child: possible underlying 
biological mechanisms. Adv Neurobiol. 2015;10:269- 83.

8. Brown S, Yelland J, Sutherland G, Baghurst P, Robinson J. Stressful life events, social health issues and low birthweight in an Australian population based birth cohort: challenges and opportunities in antenatal care. BMC Public Health. 2011;11:196.

9. Straub H, Adams M, Kim JJ, Silver RK. Antenatal depressive symptoms increase the likelihood of preterm birth. Am J Obstet Gynecol. 2012;207:329e1 -4.

10. Maternal stress and labor .K. M. Paarlberg, A. J. J. M. Vingerhoets and H. P. van Geijn. DOI: 11.3109/9781439814697-207

11. The Contribution of Maternal Stress to Preterm Birth: Issues and Considerations Pathik D. Wadhwa, MD, PhD,a,c Sonja Entringer, PhD,b,c Claudia Buss, PhD,b,c and Michael C.ClinPerinatol. 2011 Sep; 38(3): 351-384. doi: 10.1016/j.clp. 2011.06.007 